\title{
A difference-integral representation of Koornwinder polynomials
}

\author{
Eric M. Rains
}

\begin{abstract}
We construct new families of $(q-)$ difference and (contour) integral operators having nice actions on Koornwinder's multivariate orthogonal polynomials. We further show that the Koornwinder polynomials can be constructed by suitable sequences of these operators applied to the constant polynomial 1, giving the difference-integral representation of the title. Macdonald's conjectures (as proved by van Diejen and Sahi) for the principal specialization and norm follow immediately, as does a Cauchy-type identity of Mimachi.
\end{abstract}

\section{Contents}

\begin{tabular}{|lr|}
\hline $1 . \quad$ Introduction & 1 \\
\hline $2 . \quad$ Difference operators & 4 \\
\hline $3 . \quad$ Integral operators & 8 \\
\hline $4 . \quad$ The difference-integral representation & 13 \\
\hline References & 14 \\
\hline
\end{tabular}

\section{Introduction}

In 6], Koornwinder introduced a family of (symmetric) multivariate orthogonal (Laurent) polynomials orthogonal with respect to the following density on the unit torus:

$$
\begin{aligned}
& \Delta^{(n)}\left(z_{1}, z_{2}, \ldots z_{n} ; t_{0}, t_{1}, t_{2}, t_{3} ; q, t\right) \\
& \quad=\prod_{1 \leq i \leq n} \frac{\left(z_{i}^{ \pm 2} ; q\right)}{\left(t_{0} z_{i}^{ \pm 1}, t_{1} z_{i}^{ \pm 1}, t_{2} z_{i}^{ \pm 1}, t_{3} z_{i}^{ \pm 1} ; q\right)} \prod_{1 \leq i<j \leq n} \frac{\left(z_{i}^{ \pm 1} z_{j}^{ \pm 1} ; q\right)}{\left(t z_{i}^{ \pm 1} z_{j}^{ \pm 1} ; q\right)},
\end{aligned}
$$

where $(x, y, z, \ldots, w ; q)$ represents the infinite $q$-symbol

$$
\begin{aligned}
(x ; q) & :=\prod_{j \geq 0}\left(1-q^{j} x\right), \\
(x, y, z, \ldots, w ; q) & :=(x ; q)(y ; q)(z ; q) \cdots(w ; q),
\end{aligned}
$$

so in particular $\left(z_{i}^{ \pm 1} z_{j}^{ \pm 1} ; q\right)=\left(z_{i} z_{j} ; q\right)\left(z_{i} / z_{j} ; q\right)\left(z_{j} / z_{i} ; q\right)\left(1 / z_{i} z_{j} ; q\right)$.

2000 Mathematics Subject Classification. Primary 33D52; Secondary 05E35. 
To be precise, the Koornwinder polynomials $K_{\lambda}^{(n)}\left(\ldots z_{i} \ldots ; t_{0}, t_{1}, t_{2}, t_{3} ; q, t\right)$ are uniquely defined by the following requirements:

(i) $K_{\lambda}^{(n)}\left(; t_{0}, t_{1}, t_{2}, t_{3} ; q, t\right)$ is a $B C_{n}$-symmetric polynomial; i.e., a Laurent polynomial invariant under permutations of the variables and substitutions $z_{i} \mapsto z_{i}^{-1}$.

(ii) Moreover, it is monic with respect to dominance:

$$
K_{\lambda}^{(n)}\left(\ldots z_{i} \ldots ; t_{0}, t_{1}, t_{2}, t_{3} ; q, t\right)=m_{\lambda}+\text { dominated terms. }
$$

(iii) With respect to the above density, it is orthogonal to any strictly dominated monomial.

When $n=1$, Koornwinder's density becomes the following density associated to the Askey-Wilson polynomials [1]:

$$
\Delta^{(1)}\left(z ; t_{0}, t_{1}, t_{2}, t_{3} ; q, t\right)=\frac{\left(z^{ \pm 2} ; q\right)}{\left(t_{0} z^{ \pm 1}, t_{1} z^{ \pm 1}, t_{2} z^{ \pm 1}, t_{3} z^{ \pm 1} ; q\right)}
$$

and thus the Koorwinder polynomials are a multivariate analogue of Askey-Wilson polynomials, which themselves are $q$-analogues of the classical (Hermite, Laguerre, Jacobi) orthogonal polynomials.

Based on an analogy with Macdonald polynomials associated to general root systems, Macdonald made three conjectures for the Koornwinder polynomials. In addition to conjectured formulas for principal specialization

$$
k_{\lambda}^{(n)}\left(t_{0}: t_{1}, t_{2}, t_{3} ; q, t\right):=K_{\lambda}^{(n)}\left(\ldots t^{n-i} t_{0} \ldots ; t_{0}, t_{1}, t_{2}, t_{3} ; q, t\right)
$$

and for the norm with respect to the above inner product, Macdonald made a third conjecture, which we will call evaluation symmetry, stating that

$$
\frac{K_{\lambda}^{(n)}\left(\ldots q^{\mu_{i}} t^{n-i} t_{0} \ldots ; t_{0}, t_{1}, t_{2}, t_{3} ; q, t\right)}{K_{\lambda}^{(n)}\left(\ldots t^{n-i} t_{0} \ldots ; t_{0}, t_{1}, t_{2}, t_{3} ; q, t\right)}=\frac{K_{\mu}^{(n)}\left(\ldots q^{\lambda_{i}} t^{n-i} \hat{t}_{0} \ldots ; \hat{t}_{0}, \hat{t}_{1}, \hat{t}_{2}, \hat{t}_{3} ; q, t\right)}{K_{\mu}^{(n)}\left(\ldots t^{n-i} \hat{t}_{0} \ldots ; \hat{t}_{0}, \hat{t}_{1}, \hat{t}_{2}, \hat{t}_{3} ; q, t\right)}
$$

for suitably modified parameters $\hat{t}_{i}$. In [15], van Diejen showed that these conjectures were equivalent; evaluation symmetry was then proved by Sahi [14], extending work of Cherednik $\mathbf{3}$ for other root systems, using the relevant "double affine Hecke algebra" 9]; see for instance the book 7] (which treats all three conjectures directly via the double affine Hecke algebra). Essentially, this approach involves a certain large family (the affine Hecke algebra) of $q$-difference operators for which the Koornwinder polynomials are eigenfunctions; it also constructs an associated family of non-symmetric orthogonal polynomials. (A different approach, also non-symmetric and applicable to arbitrary root systems, was recently developed by Chalykh [2].)

In recent work 11, we developed a radically different approach to understanding Koornwinder polynomials (and in particular proving Macdonald's conjectures). This approach is in many respects weaker-at present, it cannot handle the nonsymmetric Koornwinder polynomials, and only works for the root system $B C_{n}$ (the hardest case for the other approaches!)-but has a significant advantage in one important respect: it can be generalized (fairly) easily to the elliptic level [12]. (See also the contributions by Gustafson and Spiridonov to this volume for discussions of related elliptic special functions.) This approach is based on Okounkov's interpolation polynomials $\mathbf{1 0}$, as well as a certain $q$-difference operator that acts nicely on these polynomials and the Koornwinder polynomials; note this operator 
is not, in fact, an element of the affine Hecke algebra, although it can presumably be constructed using the related theory of raising operators $\mathbf{7}$.

In [13, inspired by Okounkov's use of an integral operator to study and construct interpolation polynomials, we gave an explicit construction of the elliptic analogue of Koornwinder polynomials, using a sequence of difference and integral operators. There is thus a corresponding construction of Koornwinder polynomials obtained by degenerating from the elliptic case. In the present note, we describe this construction, and use it to give yet another proof of two of the three Macdonald conjectures (principal specialization and norm).

Acknowledgements. This paper is based on a talk the author gave at the Workshop on Jack, Hall-Littlewood and Macdonald Polynomials held at the International Centre for Mathematical Sciences, September 23 through 26, 2003. The author would like to thank the organizers for inviting him to that stimulating meeting, as well as the other participants for making the meeting stimulating.

Notation. Following [1], we define three multivariate analogues of $q$-symbols:

$$
\begin{aligned}
C_{\lambda}^{0}(x ; q, t) & :=\prod_{1 \leq i}\left(t^{1-i} x ; q\right)_{\lambda_{i}} \\
C_{\lambda}^{-}(x ; q, t) & :=\prod_{1 \leq i \leq j} \frac{\left(t^{j-i} x ; q\right)_{\lambda_{i}-\lambda_{j+1}}}{\left(t^{j-i} x ; q\right)_{\lambda_{i}-\lambda_{j}}} \\
C_{\lambda}^{+}(x ; q, t) & :=\prod_{1 \leq i \leq j} \frac{\left(t^{2-i-j} x ; q\right)_{\lambda_{i}+\lambda_{j}}}{\left(t^{2-i-j} x ; q\right)_{\lambda_{i}+\lambda_{j+1}}},
\end{aligned}
$$

with the usual conventions representing products of $C$ symbols via multiple arguments. We refer the reader to [11, §2] for further discussion of these symbols and the transformations they satisfy. We also follow [13 in defining two particularly important combinations of $C$ symbols:

$$
\begin{aligned}
& \Delta_{\lambda}^{0}\left(a \mid b_{1}, \ldots, b_{2 m} ; q, t\right)=\left(\frac{(q a)^{m}}{b_{1} \cdots b_{2 m}}\right)^{|\lambda|} \frac{C_{\lambda}^{0}\left(b_{1}, \ldots, b_{2 m} ; q, t\right)}{C_{\lambda}^{0}\left(q a / b_{1}, \ldots, q a / b_{2 m} ; q, t\right)} \\
& \Delta_{\lambda}\left(a \mid b_{1}, \ldots, b_{2 m} ; q, t\right)=\Delta_{\lambda}^{0}\left(a \mid b_{1}, \ldots, b_{2 m} ; q, t\right) \frac{t^{2 n(\lambda)}(t / q a)^{|\lambda|} C_{2 \lambda^{2}}^{0}(q a ; q, t)}{C_{\lambda}^{-}(q, t ; q, t) C_{\lambda}^{+}(a, q a / t ; q, t)}
\end{aligned}
$$

(These are, of course, limits of the corresponding symbols of [13 appropriate to the Koornwinder degeneration.)

Given a partition $\lambda$ with at most $n$ parts, the $B C_{n}$-symmetric monomial function $m_{\lambda}$ is defined to be the symmetrization of the monomial $\prod_{i} z_{i}^{\lambda_{i}}$. Note that in terms of the usual monomial symmetric function, we have $m_{\lambda}\left(\ldots z_{i} \ldots\right)=$ $m_{\lambda}\left(\ldots z_{i}+z_{i}^{-1} \ldots\right)$. We define a $B C_{n}$-symmetric function $e_{\lambda}$ analogously, for partitions with $\lambda_{1} \leq n$.

If $f$ is a $B C_{n}$-symmetric polynomial, we define

$$
\begin{gathered}
\langle f\rangle_{t_{0}, t_{1}, t_{2}, t_{3} ; q, t}^{\prime(n)}=\frac{(q ; q)^{n}}{(t ; q)^{n} 2^{n} n !} \int f\left(\ldots z_{i} \ldots\right) \Delta^{(n)}\left(\ldots z_{i} \ldots ; t_{0}, t_{1}, t_{2}, t_{3} ; q, t\right) \\
\prod_{1 \leq i \leq n} \frac{d z_{i}}{2 \pi \sqrt{-1} z_{i}},
\end{gathered}
$$


and

$$
\langle f\rangle_{t_{0}, t_{1}, t_{2}, t_{3} ; q, t}^{(n)}=\frac{\langle f\rangle_{t_{0}, t_{1}, t_{2}, t_{3} ; q, t}^{\prime(n)}}{\langle 1\rangle_{t_{0}, t_{1}, t_{2}, t_{3} ; q, t}^{\prime(n)}}
$$

surpressing $(n)$ when it follows from context. If $\left|t_{0}\right|,\left|t_{1}\right|,\left|t_{2}\right|,\left|t_{3}\right|,|q|,|t|<1$, then the contour of integration will be the unit torus; otherwise, the contour needs to be modified to meromorphically continue from this case. We finally define

$$
N_{\lambda}^{(n)}\left(t_{0}: t_{1}, t_{2}, t_{3} ; q, t\right):=\frac{\left\langle K_{\lambda}^{(n)}\left(; t_{0}, t_{1}, t_{2}, t_{3} ; q, t\right) K_{\lambda}^{(n)}\left(; t_{0}, t_{1}, t_{2}, t_{3} ; q, t\right)\right\rangle_{t_{0}, t_{1}, t_{2}, t_{3} ; q, t}}{k_{\lambda}^{(n)}\left(t_{0}: t_{1}, t_{2}, t_{3} ; q, t\right) k_{\lambda}^{(n)}\left(t_{0}: t_{1}, t_{2}, t_{3} ; q, t\right)}
$$

\section{Difference operators}

Of course, the first thing to consider when studying a nice family of orthogonal polynomials is the normalization of the inner product density itself. In the case of the Koornwinder polynomials, this normalization was given by the following theorem of Gustafson.

Theorem 2.1. 4] For arbitrary complex parameters $q, t, t_{0}, t_{1}, t_{2}, t_{3}$ all of absolute value less than 1 ,

$$
\langle 1\rangle_{t_{0}, t_{1}, t_{2}, t_{3} ; q, t}^{(n)}=\prod_{1 \leq j \leq n} \frac{\left(t^{2 n-j-1} t_{0} t_{1} t_{2} t_{3} ; q\right)}{\left(t^{n-j+1} ; q\right) \prod_{0 \leq r<s \leq 3}\left(t^{n-j} t_{r} t_{s} ; q\right)} .
$$

We will discuss Gustafson's proof in the sequel, but for the present the following proof will be more relevant. First, a lemma.

Lemma 2.2. For arbitrary complex numbers $t, t_{0}, t_{1}$,

$\prod_{1 \leq i \leq n}\left(1+R\left(z_{i}\right)\right) \prod_{1 \leq i \leq n} \frac{\left(1-t_{0} z_{i}\right)\left(1-t_{1} z_{i}\right)}{1-z_{i}^{2}} \prod_{1 \leq i<j \leq n} \frac{1-t z_{i} z_{j}}{1-z_{i} z_{j}}=\prod_{1 \leq i \leq n}\left(1-t^{n-i} t_{0} t_{1}\right)$,

where $R(z)$ is the operator defined by $R(z) f(z)=f(1 / z)$.

Proof. If we multiply the left-hand side by the fully antisymmetric polynomial

$$
\Delta(z)=\prod_{1 \leq i \leq n}\left(z_{i}-1 / z_{i}\right) \prod_{1 \leq i<j \leq n}\left(z_{i}+1 / z_{i}-z_{j}-1 / z_{j}\right),
$$

we obtain the sum

$$
\prod_{1 \leq i \leq n}\left(1-R\left(z_{i}\right)\right) \prod_{1 \leq i \leq n} \frac{-\left(1-t_{0} z_{i}\right)\left(1-t_{1} z_{i}\right)}{z_{i}} \prod_{1 \leq i<j \leq n} \frac{\left(1-t z_{i} z_{j}\right)\left(z_{j}-z_{i}\right)}{z_{i} z_{j}} .
$$

Since each term is a Laurent polynomial, the sum is itself a Laurent polynomial. Moreover, since

$$
\prod_{1 \leq i \leq n} \frac{-\left(1-t_{0} z_{i}\right)\left(1-t_{1} z_{i}\right)}{z_{i}} \prod_{1 \leq i<j \leq n} \frac{\left(1-t z_{i} z_{j}\right)\left(z_{j}-z_{i}\right)}{z_{i} z_{j}}
$$

is antisymmetric under permutations of the variables, and the group generated by the $R\left(z_{i}\right)$ is normalized by $S_{n}$, it follows that the sum will also be antisymmetric. Since it is also antisymmetric under each $R\left(z_{i}\right)$, we find that it is antisymmetric 
under the full action of $B C_{n}$. But then it must be a multiple of $\Delta(z)$. Comparing degrees, we find that the original left-hand side sums to a constant.

To compute this constant, we can proceed in either of two ways. First, if we specialize $z_{i}=t^{n-i} t_{0}$, only one term on the left survives, which immediately simplifies to give the desired result. Alternatively, we can simply compute the coefficient of the leading monomial of

$$
\prod_{1 \leq i \leq n}\left(1-R\left(z_{i}\right)\right) \prod_{1 \leq i \leq n} \frac{-\left(1-t_{0} z_{i}\right)\left(1-t_{1} z_{i}\right)}{z_{i}} \prod_{1 \leq i<j \leq n} \frac{\left(1-t z_{i} z_{j}\right)\left(z_{j}-z_{i}\right)}{z_{i} z_{j}}
$$

We can now give the associated proof of Theorem 2.1]

Proof. Factor the integrand as

$$
\begin{aligned}
\Delta^{(n)}\left(\ldots z_{i} \ldots ; t_{0}, t_{1}, t_{2}, t_{3} ; q, t\right)= & \Delta_{+}^{(n)}\left(\ldots z_{i} \ldots ; t_{0}, t_{1}, t_{2}, t_{3} ; q, t\right) \\
& \Delta_{+}^{(n)}\left(\ldots z_{i}^{-1} \ldots ; t_{0}, t_{1}, t_{2}, t_{3} ; q, t\right),
\end{aligned}
$$

where

$$
\Delta_{+}^{(n)}\left(\ldots z_{i} \ldots ; t_{0}, t_{1}, t_{2}, t_{3} ; q, t\right)=\prod_{1 \leq i \leq n} \frac{\left(z_{i}^{2} ; q\right)}{\left(t_{0} z_{i}, t_{1} z_{i}, t_{2} z_{i}, t_{3} z_{i} ; q\right)} \prod_{1 \leq i<j \leq n} \frac{\left(z_{i} z_{j}^{ \pm 1} ; q\right)}{\left(t z_{i} z_{j}^{ \pm 1} ; q\right)}
$$

and consider the integral

$$
\begin{aligned}
& \int \Delta_{+}^{(n)}\left(\ldots q^{1 / 2} z_{i} \ldots ; t_{0}^{\prime}, t_{1}^{\prime}, t_{2}^{\prime}, t_{3}^{\prime} ; q, t\right) \\
& \quad \Delta_{+}^{(n)}\left(\ldots z_{i}^{-1} \ldots ; t_{0}, t_{1}, t_{2}, t_{3} ; q, t\right) \prod_{1 \leq i \leq n} \frac{d z_{i}}{2 \pi \sqrt{-1} z_{i}}
\end{aligned}
$$

where

$$
\left(t_{0}^{\prime}, t_{1}^{\prime}, t_{2}^{\prime}, t_{3}^{\prime}\right)=\left(q^{1 / 2} t_{0}, q^{1 / 2} t_{1}, q^{-1 / 2} t_{2}, q^{-1 / 2} t_{3}\right) .
$$

Now, Lemma 2.2 can be expressed in the equivalent form

$$
\prod_{1 \leq i \leq n}\left(1+R\left(z_{i}\right)\right) \frac{\Delta_{+}^{(n)}\left(\ldots q^{1 / 2} z_{i} \ldots ; t_{0}^{\prime}, t_{1}^{\prime}, t_{2}^{\prime}, t_{3}^{\prime} ; q, t\right)}{\Delta_{+}^{(n)}\left(\ldots z_{i} \ldots ; t_{0}, t_{1}, t_{2}, t_{3} ; q, t\right)}=\prod_{1 \leq i \leq n}\left(1-t^{n-i} t_{0} t_{1}\right)
$$

and we thus conclude that

$$
\begin{aligned}
& \int \Delta_{+}^{(n)}\left(\ldots q^{1 / 2} z_{i} \ldots ; t_{0}^{\prime}, t_{1}^{\prime}, t_{2}^{\prime}, t_{3}^{\prime} ; q, t\right) \\
& \Delta_{+}^{(n)}\left(\ldots z_{i}^{-1} \ldots ; t_{0}, t_{1}, t_{2}, t_{3} ; q, t\right) \prod_{1 \leq i \leq n} \frac{d z_{i}}{2 \pi \sqrt{-1} z_{i}} \\
& =\frac{(t ; q)^{n} n !}{(q ; q)^{n}} \prod_{1 \leq i \leq n}\left(1-t^{n-i} t_{0} t_{1}\right)\langle 1\rangle_{t_{0}, t_{1}, t_{2}, t_{3} ; q, t}^{\prime(n)}
\end{aligned}
$$

If we apply the change of variables $z_{i} \mapsto q^{-1 / 2} / z_{i}$, we obtain a similar simplification; we thus conclude

$$
\langle 1\rangle_{t_{0}, t_{1}, t_{2}, t_{3} ; q, t}^{\prime(n)}=\prod_{1 \leq i \leq n}\left(\frac{1-t^{n-i} t_{2} t_{3} / q}{1-t^{n-i} t_{0} t_{1}}\right)\langle 1\rangle_{q^{1 / 2} t_{0}, q^{1 / 2} t_{1}, q^{-1 / 2} t_{2}, q^{-1 / 2} t_{3} ; q, t}^{\prime(n)}
$$


Since the desired right-hand side satisfies the same recurrence, and both sides are invariant under permutations of $t_{0}$ through $t_{3}$, we conclude that the ratio of the two sides of the desired identity is a function only of $t_{0} t_{1} t_{2} t_{3}, q$, and $t$.

We can then compute this ratio by expanding the limiting case $t^{n-1} t_{2} t_{3}=1$ via residue calculus.

The key observation is that this proof can be viewed as being based on adjointness of difference operators. We define three $q$-difference operators as follows.

Definition 1 . Let $t_{0}, t_{1}, t_{2}, t_{3}, q, t$ be arbitrary parameters, and define difference operators acting on $B C_{n}$-symmetric polynomials as follows:

$$
\begin{gathered}
\left(D_{q}^{-(n)}(t) f\right)\left(\ldots z_{i} \ldots\right)=\prod_{1 \leq i \leq n}\left(1+R\left(z_{i}\right)\right) \prod_{1 \leq i \leq n} \frac{z_{i}}{1-z_{i}^{2}} \\
\prod_{1 \leq i<j \leq n} \frac{1-t z_{i} z_{j}}{1-z_{i} z_{j}} f\left(\ldots \sqrt{q} z_{i} \ldots\right) \\
\left(D_{q}^{(n)}\left(t_{0}, t_{1} ; t\right) f\right)\left(\ldots z_{i} \ldots\right)=\prod_{1 \leq i \leq n}\left(1+R\left(z_{i}\right)\right) \prod_{1 \leq i \leq n} \frac{\left(1-t_{0} z_{i}\right)\left(1-t_{1} z_{i}\right)}{1-z_{i}^{2}} \\
\prod_{1 \leq i<j \leq n} \frac{1-t z_{i} z_{j}}{1-z_{i} z_{j}} f\left(\ldots \sqrt{q} z_{i} \ldots\right) \\
\left(D_{q}^{+(n)}\left(t_{0}, t_{1}, t_{2}, t_{3} ; t\right) f\right)\left(\ldots z_{i} \ldots\right) \\
=\prod_{1 \leq i \leq n}\left(1+R\left(z_{i}\right)\right) \prod_{1 \leq i \leq n} \frac{\left(1-t_{0} z_{i}\right)\left(1-t_{1} z_{i}\right)\left(1-t_{2} z_{i}\right)\left(1-t_{3} z_{i}\right)}{z_{i}\left(1-z_{i}^{2}\right)} \\
\prod_{1 \leq i<j \leq n} \frac{1-t z_{i} z_{j}}{1-z_{i} z_{j}} f\left(\ldots \sqrt{q} z_{i} \ldots\right)
\end{gathered}
$$

THEOREM 2.3. The above difference operators take $B C_{n}$-symmetric polynomials to $B C_{n}$-symmetric polynomials, acting triangularly with respect to dominance of monomials:

$$
\begin{aligned}
D_{q}^{-(n)}(t) m_{\lambda} & =q^{-|\lambda| / 2} \prod_{1 \leq i \leq n}\left(1-q^{\lambda_{i}} t^{n-i}\right) m_{\lambda-1^{n}}+\text { dominated terms } \\
D_{q}^{(n)}\left(t_{0}, t_{1} ; t\right) m_{\lambda} & =q^{-|\lambda| / 2} \prod_{1 \leq i \leq n}\left(1-q^{\lambda_{i}} t^{n-i} t_{0} t_{1}\right) m_{\lambda}+\text { dominated terms }
\end{aligned}
$$

$$
\begin{aligned}
& D_{q}^{+(n)}\left(t_{0}, t_{1}, t_{2}, t_{3} ; t\right) m_{\lambda}=q^{-|\lambda| / 2} \prod_{1 \leq i \leq n}\left(1-q^{\lambda_{i}} t^{n-i} t_{0} t_{1} t_{2} t_{3}\right) m_{\lambda+1^{n}} \\
& + \text { dominated terms. }
\end{aligned}
$$

Furthermore, they satisfy the following adjointness relations with respect to the Koornwinder inner product:

$$
\left\langle f D_{q}^{(n)}\left(t_{0}, t_{1} ; t\right) g\right\rangle_{t_{0}, t_{1}, t_{2}, t_{3} ; q, t}^{\prime(n)}=\left\langle g D_{q}^{(n)}\left(t_{2}^{\prime}, t_{3}^{\prime} ; t\right) f\right\rangle_{t_{0}^{\prime}, t_{1}^{\prime}, t_{2}^{\prime}, t_{3}^{\prime} ; q, t}^{\prime(n)},
$$

where $\left(t_{0}^{\prime}, t_{1}^{\prime}, t_{2}^{\prime}, t_{3}^{\prime}\right)=\left(q^{1 / 2} t_{0}, q^{1 / 2} t_{1}, q^{-1 / 2} t_{2}, q^{-1 / 2} t_{3}\right)$, and

$$
\left\langle f D_{q}^{+(n)}\left(t_{0}, t_{1}, t_{2}, t_{3} ; t\right) g\right\rangle_{t_{0}, t_{1}, t_{2}, t_{3} ; q, t}^{\prime(n)}=q^{n / 2}\left\langle g D_{q}^{-(n)}(t) f\right\rangle_{q^{1 / 2} t_{0}, q^{1 / 2} t_{1}, q^{1 / 2} t_{2}, q^{1 / 2} t_{3} ; q, t}^{\prime(n)}
$$


Proof. The same argument used to prove Theorem 2.1] extends immediately to give adjointness. Similarly, that the operators take polynomials to polynomials follows as in the proof of Lemma 2.2. For instance, for $D^{-}$, we find that after clearing the denominator, we obtain $\prod_{i}\left(1-R\left(z_{i}\right)\right)$ applied to a Laurent polynomial in which every monomial is dominated by $\prod_{i} z_{i}^{\lambda_{i}+n-i}$. When we antisymmetrize and divide the denominator back out, we thus find that every monomial of the result is dominated by $\prod_{i} z_{i}^{\lambda_{i}-1}$ as required. Moreover, the coefficient of that monomial is readily computed as given above. (See, for instance, 11, Theorem 3.1].)

COROLlaRY 2.4. The difference operators act on Koornwinder polynomials as follows.

$$
\begin{aligned}
& D_{q}^{-(n)}(t) K_{\lambda}^{(n)}\left(; q^{-1 / 2} t_{0}, q^{-1 / 2} t_{1}, q^{-1 / 2} t_{2}, q^{-1 / 2} t_{3} ; q, t\right) \\
& =q^{-|\lambda| / 2} \prod_{1 \leq i \leq n}\left(1-q^{\lambda_{i}} t^{n-i}\right) K_{\lambda-1^{n}}^{(n)}\left(; t_{0}, t_{1}, t_{2}, t_{3} ; q, t\right) \\
& D_{q}^{(n)}\left(t_{0}, t_{1} ; t\right) K_{\lambda}^{(n)}\left(; q^{1 / 2} t_{0}, q^{1 / 2} t_{1}, q^{-1 / 2} t_{2}, q^{-1 / 2} t_{3} ; q, t\right) \\
& =q^{-|\lambda| / 2} \prod_{1 \leq i \leq n}\left(1-q^{\lambda_{i}} t^{n-i} t_{0} t_{1}\right) K_{\lambda}^{(n)}\left(; t_{0}, t_{1}, t_{2}, t_{3} ; q, t\right) \\
& \begin{array}{l}
D_{q}^{+(n)}\left(t_{0}, t_{1}, t_{2}, t_{3} ; t\right) K_{\lambda}^{(n)}\left(; q^{1 / 2} t_{0}, q^{1 / 2} t_{1}, q^{1 / 2} t_{2}, q^{1 / 2} t_{3} ; q, t\right) \\
\quad=q^{-|\lambda| / 2} \prod_{1 \leq i \leq n}\left(1-q^{\lambda_{i}} t^{n-i} t_{0} t_{1} t_{2} t_{3}\right) K_{\lambda+1^{n}}^{(n)}\left(; t_{0}, t_{1}, t_{2}, t_{3} ; q, t\right)
\end{array}
\end{aligned}
$$

REMARK. Given this action on Koornwinder polynomials, it is natural to wonder how our difference operators relate to the theory of double affine Hecke algebras. The operator $D_{q}^{(n)}$ certainly has such an interpretation, as follows. There is a diagram automorphism of the root system $B C_{n}$ which gives rise to an outer automorphism of its Weyl group; using this in the standard way gives an operator corresponding to translation by the (miniscule) weight $\left(\frac{1}{2}, \ldots \frac{1}{2}\right)$ that commutes (modulo a parameter shift) with the usual commutative subalgebra. Symmetrizing this gives a difference operator which, by comparing actions on Koornwinder polynomials, must equal $D_{q}^{(n)}$. Most likely, the operators $D_{q}^{+(n)}$ and $D_{q}^{-(n)}$ arise similarly, as analogues of "shift operators" (see [7, §5.9] for the usual version).

In particular, we see that $D^{-}$acts as a lowering operator, and $D^{+}$acts as a raising operator. Moreover, it is clear that we can combine these "first-order" operators in eight different ways to obtain "second-order" operators for which the Koornwinder polynomials are actually eigenfunctions. These second-order operators all lie in the center of the affine Hecke algebra; the first-order operators do not, but can presumably still be obtained from that theory.

For our present purposes, the main consequence of this action is the following recurrences for the principal specialization and the norm:

COROLlary 2.5. For the principal specialization, we have:

$$
\frac{k_{\lambda}^{(n)}\left(t_{0}: t_{1}, t_{2}, t_{3} ; q, t\right)}{k_{\lambda}^{(n)}\left(q^{1 / 2} t_{0}: q^{1 / 2} t_{1}, q^{-1 / 2} t_{2}, q^{-1 / 2} t_{3} ; q, t\right)}=q^{|\lambda| / 2} \prod_{1 \leq i \leq n} \frac{1-t^{n-i} t_{0} t_{1}}{1-q^{\lambda_{i}} t^{n-i} t_{0} t_{1}}
$$


and

$$
\begin{aligned}
& \frac{k_{\lambda+1^{n}}^{(n)}\left(t_{0}: t_{1}, t_{2}, t_{3} ; q, t\right)}{k_{\lambda}^{(n)}\left(q^{1 / 2} t_{0}: q^{1 / 2} t_{1}, q^{1 / 2} t_{2}, q^{1 / 2} t_{3} ; q, t\right)} \\
& \quad=q^{|\lambda| / 2} \prod_{1 \leq i \leq n} \frac{\left(1-t^{n-i} t_{0} t_{1}\right)\left(1-t^{n-i} t_{0} t_{2}\right)\left(1-t^{n-i} t_{0} t_{3}\right)}{t^{n-i} t_{0}\left(1-q^{\lambda_{i}} t^{n-i} t_{0} t_{1} t_{2} t_{3}\right)}
\end{aligned}
$$

For the norms of (normalized) Koornwinder polynomials, we have:

$$
\begin{aligned}
& \frac{N_{\lambda}^{(n)}\left(t_{0}: t_{1}, t_{2}, t_{3} ; q, t\right)}{N_{\lambda}^{(n)}\left(q^{1 / 2} t_{0}: q^{1 / 2} t_{1}, q^{-1 / 2} t_{2}, q^{-1 / 2} t_{3} ; q, t\right)} \\
& \quad=\prod_{1 \leq i \leq n} \frac{q^{-\lambda_{i}}\left(1-q^{\lambda_{i}} t^{n-i} t_{0} t_{1}\right)\left(1-q^{\lambda_{i}} t^{n-i} t_{2} t_{3} / q\right)}{\left(1-t^{n-i} t_{0} t_{1}\right)\left(1-t^{n-i} t_{2} t_{3} / q\right)},
\end{aligned}
$$

and

$$
\begin{aligned}
\frac{N_{\left(\lambda+1^{n}\right)}^{(n)}\left(t_{0}: t_{1}, t_{2}, t_{3} ; q, t\right)}{N_{\lambda}^{(n)}\left(q^{1 / 2} t_{0}: q^{1 / 2} t_{1}, q^{1 / 2} t_{2}, q^{1 / 2} t_{3} ; q, t\right)} \\
=t_{0}^{2 n} t^{n(n-1)} \prod_{1 \leq i \leq n} \frac{\left(1-t^{n-i} t_{1} t_{2}\right)\left(1-t^{n-i} t_{1} t_{3}\right)\left(1-t^{n-i} t_{2} t_{3}\right)}{\left(1-t^{n-i} t_{0} t_{1}\right)\left(1-t^{n-i} t_{0} t_{2}\right)\left(1-t^{n-i} t_{0} t_{3}\right)} \\
\prod_{1 \leq i \leq n} \frac{q^{-\lambda_{i}}\left(1-q^{\lambda_{i}} t^{n-i} q\right)\left(1-q^{\lambda_{i}} t^{n-i} t_{0} t_{1} t_{2} t_{3}\right)}{\left(1-t^{2 n-i-1} t_{0} t_{1} t_{2} t_{3}\right)\left(1-q t^{2 n-i-1} t_{0} t_{1} t_{2} t_{3}\right)} .
\end{aligned}
$$

Proof. For the first two recurrence relations, we observe that $D_{q}^{(n)}\left(t_{0}, t_{1} ; t\right)$ and $D_{q}^{+(n)}\left(t_{0}, t_{1}, t_{2}, t_{3} ; t\right)$ respect principal specialization (relative to $t_{0}$ ), and thus these relations follow immediately from the action of these operators on Koornwinder polynomials. Similarly, the norm recurrence follows from this action together with adjointness.

These recurrences are not quite enough to completely specify these quantities; there is still freedom when $\lambda_{n}=0$ to multiply by an arbitrary function of $t_{0} t_{1} t_{2} t_{3}, q$, and $t$. To eliminate this freedom, we will use another, dual, collection of recurrences.

\section{Integral operators}

Gustafson's original proof of Theorem [2.1 was based on the following integral identity.

Theorem 3.1. [5] For any integer $n \geq 0$, choose complex parameters $q, t_{0}, \ldots$, $t_{2 n+1},|q|<1$, such that the sets

$$
\left\{q^{k} t_{r}: k \geq 0,0 \leq r \leq 2 n+1\right\} \text { and }\left\{q^{-k} / t_{r}: k \geq 0,0 \leq r \leq 2 n+1\right\}
$$

are disjoint, and thus one can choose a contour $C$ containing the first set and excluding the second set. Then

$$
\begin{array}{r}
\frac{(q ; q)^{n}}{2^{n} n !} \int_{C^{n}} \prod_{1 \leq i<j \leq n}\left(z_{i}^{ \pm 1} z_{j}^{ \pm 1} ; q\right) \prod_{1 \leq i \leq n} \frac{\left(z_{i}^{ \pm 2} ; q\right)}{\prod_{0 \leq r<2 n+2}\left(t_{r} z_{i}^{ \pm 1} ; q\right)} \frac{d z_{i}}{2 \pi \sqrt{-1} z_{i}} \\
=\frac{\left(t_{0} t_{1} \cdots t_{2 n+1} ; q\right)}{\prod_{0 \leq r<s \leq 2 n+1}\left(t_{r} t_{s} ; q\right)}
\end{array}
$$


REMARK. In addition to the proof in [5] \$7], based on a multivariate bilateral hypergeometric summation identity, and a proof along the lines of $\mathbf{1 3}$ using the fact that when $n$ pairs of parameters multiply to $q$, the result is a determinant of Askey-Wilson integrals, we remark that there is a third proof based on the identity

$$
\prod_{1 \leq i \leq n}\left(1+R\left(z_{i}\right)\right) \frac{\left(1-t_{0} z_{i}\right) \cdots\left(1-t_{n} z_{i}\right)}{1-z_{i}^{2}} \prod_{1 \leq i<j \leq n} \frac{1}{1-z_{i} z_{j}}=\prod_{0 \leq i<j \leq n}\left(1-t_{i} t_{j}\right)
$$

which gives an argument along the lines of our proof of Theorem 2.1 above. As in that case, this gives rise to pairs of adjoint difference operators acting on $B C_{n^{-}}$ symmetric polynomials; it is not clear, however, what significance these operators might have.

Gustafson's proof of Theorem [2.1 is based on the following double integral:

$$
\begin{array}{r}
\int_{C^{n+1}} \int_{C^{\prime n}} \frac{\prod_{0 \leq i<j \leq n}\left(x_{i}^{ \pm 1} x_{j}^{ \pm 1} ; q\right) \prod_{1 \leq i<j \leq n}\left(y_{i}^{ \pm 1} y_{j}^{ \pm 1} ; q\right)}{\prod_{\substack{0 \leq i \leq n \\
1 \leq j \leq n}}\left(\sqrt{t} x_{i}^{ \pm 1} y_{j}^{ \pm 1} ; q\right)} \prod_{1 \leq i \leq n}\left(y_{i}^{ \pm 2} ; q\right) \frac{d y_{i}}{2 \pi \sqrt{-1} y_{i}} \\
\prod_{0 \leq i \leq n} \frac{\left(x_{i}^{ \pm 2} ; q\right)}{\prod_{0 \leq r \leq 3}\left(t_{r} x_{i}^{ \pm 1} ; q\right)} \frac{d x_{i}}{2 \pi \sqrt{-1} x_{i}}
\end{array}
$$

with appropriate choices of contour. Both the $x$ and $y$ variables independently can be integrated out via Theorem 3.1 the resulting identity gives a recurrence in $n$ for the Koornwinder normalization, from which Theorem 2.1 follows immediately.

Just as the first proof above gives rise to adjoint pairs of difference operators, Gustafson's proof gives rise to adjoint pairs of integral operators. Defining the operators and proving adjointness is straightforward; the main difficulty is simply proving that they take $B C_{n}$-symmetric polynomials to $B C_{n}$-symmetric polynomials. The key fact is the following generalization of Theorem 3.1 Define an integral operator $I^{*(n)}(q)$ taking $B C_{n}$-symmetric polynomials to $S_{2 n+2}$-symmetric functions by

$$
\left(I^{*(n)}(q) f\right)\left(t_{0}, t_{1}, \ldots, t_{2 n+1}\right)=\int_{C^{n}} f\left(\ldots z_{i} \ldots\right) \kappa\left(\ldots z_{i} \ldots\right) \frac{d z_{i}}{2 \pi \sqrt{-1} z_{i}}
$$

where

$$
\begin{aligned}
\kappa\left(\ldots z_{i} \ldots\right)= & \frac{\prod_{0 \leq r<s \leq 2 n+1}\left(t_{r} t_{s} ; q\right)}{\left(t_{0} t_{1} \cdots t_{2 n+1} ; q\right)} \frac{(q ; q)^{n}}{2^{n} n !} \\
& \prod_{1 \leq i<j \leq n}\left(z_{i}^{ \pm 1} z_{j}^{ \pm 1} ; q\right) \prod_{1 \leq i \leq n} \frac{\left(z_{i}^{ \pm 2} ; q\right)}{\prod_{0 \leq r<2 n+2}\left(t_{r} z_{i}^{ \pm 1} ; q\right)}
\end{aligned}
$$

and the contour $C$ is as above.

Theorem 3.2. If

$$
f\left(\ldots z_{i} \ldots\right)=\prod_{\substack{1 \leq i \leq n \\ 1 \leq j \leq m}}\left(y_{j}+y_{j}^{-1}-z_{i}-z_{i}^{-1}\right),
$$


then

$$
\begin{aligned}
\left(I^{*(n)}(q) f\right)\left(t_{0}, t_{1}, \ldots, t_{2 n+1}\right)= & \left(t_{0} t_{1} \cdots t_{2 n+1} ; q\right)_{m}^{-1} \\
& \prod_{1 \leq i \leq m}\left(1+R\left(y_{i}\right)\right) \frac{\prod_{0 \leq r<2 n+2}\left(1-t_{r} y_{i}\right)}{y_{i}^{n}\left(1-y_{i}^{2}\right)} \\
& \prod_{1 \leq i<j \leq m} \frac{1-q y_{i} y_{j}}{1-y_{i} y_{j}} .
\end{aligned}
$$

Proof. The key step is the following lemma.

Lemma 3.3. For any $B C_{n}$-symmetric polynomial $f$,

$$
\begin{aligned}
(1-R(y)) y^{-n} & \prod_{0 \leq r<2 n}\left(1-t_{r} y\right)\left(I^{*(n)}(q) f\right)\left(t_{0}, t_{1}, \ldots, t_{2 n-1}, q y, 1 / y\right) \\
& =\left(1-t_{0} t_{1} \ldots t_{2 n-1}\right) y^{-1}\left(1-y^{2}\right)\left(I^{*(n-1)}(q) \tilde{f}\right)\left(t_{0}, t_{1}, \ldots, t_{2 n-1}\right),
\end{aligned}
$$

where

$$
\tilde{f}\left(z_{1}, \ldots, z_{n-1}\right)=\prod_{1 \leq i<n}\left(y+1 / y-z_{i}-1 / z_{i}\right) f\left(z_{1}, \ldots, z_{n-1}, y\right)
$$

Proof. In fact, the two integrals on the left have exactly the same integrand, and thus their difference is controlled entirely by the difference in contours. This difference is simply that one contour contains $y$ and excludes $1 / y$, while the other contains $1 / y$ and excludes $y$. We can thus expand the left-hand side via residue calculus; the result follows.

In particular, the case $(n, m)$ of the theorem implies the case $(n-1, m+1)$; since the case $m=0$ is just Theorem 3.1 the result follows.

Note that aside from the factor $\left(t_{0} t_{1} \cdots t_{2 n+1} ; q\right)_{m}^{-1}$, the right-hand side is polynomial in $t_{0}, \ldots, t_{2 n+1}$, and thus the following three integral operators take $B C_{n^{-}}$ symmetric polynomials to $B C_{n^{\prime}}$ symmetric polynomials, for $n^{\prime}=n+1, n$, or $n-1$ as appropriate.

Definition 2. Define three integral operators acting on $B C_{n}$-symmetric polynomials as follows.

$$
\begin{aligned}
\left(I_{t}^{+(n)}(q) f\right)\left(z_{1}, \ldots, z_{n+1}\right) & =\left(I_{t}^{*(n)}(q) f\right)\left(\ldots \sqrt{t} z_{i}^{ \pm 1} \ldots\right) \\
\left(I_{t}^{(n)}\left(t_{0}, t_{1} ; q\right) f\right)\left(z_{1}, \ldots, z_{n}\right) & =\left(I_{t}^{*(n)}(q) f\right)\left(t_{0}, t_{1}, \ldots \sqrt{t} z_{i}^{ \pm 1} \ldots\right)
\end{aligned}
$$

$$
\left(I_{t}^{-(n)}\left(t_{0}, t_{1}, t_{2}, t_{3} ; q\right) f\right)\left(z_{1}, \ldots, z_{n-1}\right)=\left(I_{t}^{*(n)}(q) f\right)\left(t_{0}, t_{1}, t_{2}, t_{3}, \ldots \sqrt{t} z_{i}^{ \pm 1} \ldots\right) .
$$

THEOREM 3.4. The above operators act on ( $B C_{n}$-symmetric) monomials as follows.

$$
I_{t}^{+(n)}(q) m_{\lambda}^{(n)}=t^{|\lambda| / 2} \prod_{1 \leq i \leq m} \frac{1-t^{n+1-\lambda_{i}^{\prime}} q^{i-1}}{1-t^{n+1} q^{i-1}} m_{\lambda}^{(n+1)}+\text { dominated terms }
$$

$$
I_{t}^{(n)}\left(t_{0}, t_{1} ; q\right) m_{\lambda}^{(n)}=t^{|\lambda| / 2} \prod_{1 \leq i \leq m} \frac{1-t^{n-\lambda_{i}^{\prime}} q^{i-1} t_{0} t_{1}}{1-t^{n} q^{i-1} t_{0} t_{1}} m_{\lambda}^{(n)}+\text { dominated terms, }
$$




$$
\begin{aligned}
I_{t}^{-(n)}\left(t_{0}, t_{1}, t_{2}, t_{3} ; q\right) m_{\lambda}^{(n)}= & t^{|\lambda| / 2} \prod_{\substack{1 \leq i \leq m \\
1 \leq t^{-1} q^{i-1} t_{0} t_{1} t_{2} t_{3}}} \frac{1-t^{n-1-\lambda_{i}^{\prime}} m^{i-1} t_{1} t_{2} t_{3}}{\left.1-t^{n-1}\right)} \\
& + \text { dominated terms }
\end{aligned}
$$

where $m_{\lambda}^{(n)}:=0$ if $\ell(\lambda)>n$.

Proof. We first observe that our integral operators are in fact very closely related to our difference operators; an integral operator acting on the $z$ variables of a product

$$
f_{n, m}=\prod_{1 \leq i \leq n, 1 \leq j \leq m}\left(y_{j}+1 / y_{j}-z_{i}-1 / z_{i}\right)
$$

becomes a difference operator acting on the $y$ variables of a corresponding product. More precisely, we have the following special cases of Theorem 3.2

$$
I_{t}^{(n)}\left(t_{0}, t_{1} ; q\right)_{z} f_{n, m}=\left(t^{n} t_{0} t_{1} ; q\right)_{m}^{-1} t^{m n / 2} D_{t}^{(m)}\left(t_{0}, t_{1} ; q\right)_{y} f_{n, m}
$$

$$
I_{t}^{-(n)}\left(t_{0}, t_{1} ; q\right)_{z} f_{n, m}=\left(t^{n-1} t_{0} t_{1} t_{2} t_{3} ; q\right)_{m}^{-1} t^{m(n-1) / 2} D_{t}^{+(m)}\left(t_{0}, t_{1}, t_{2}, t_{3} ; q\right)_{y} f_{n-1, m} .
$$

Now, the product $f_{n, m}$ behaves nicely with respect to dominance of monomials: we have an expansion

$$
f_{n, m}=\sum_{\lambda \subset m^{n}}(-1)^{|\lambda|} m_{\lambda}\left(z_{1}, \ldots, z_{n}\right) m_{n^{m}-\lambda^{\prime}}\left(y_{1}, \ldots, y_{m}\right)+\text { dominated terms }
$$

in the sense that the coefficient of $m_{\lambda}(z)$ has dominant monomial $(-1)^{|\lambda|} m_{n^{m}-\lambda^{\prime}}(y)$, and vice versa. (Note that $n^{m}-\lambda^{\prime}$ dominates $\mu$ if and only if $m^{n}-\mu^{\prime}$ dominates $\lambda$, so this condition is indeed symmetrical.)

Thus the fact that the difference operators are triangular implies that the integral operators are triangular, and similarly for determining the diagonal coefficients; the theorem follows.

LEMMA 3.5. The integral operators satisfy the adjointness relations

$$
\left\langle g I_{t}^{(n)}\left(t_{0}, t_{1} ; q\right) f\right\rangle_{t_{0}^{\prime}, t_{1}^{\prime}, t_{2}^{\prime}, t_{3}^{\prime} ; q, t}^{(n)}=\left\langle f I_{t}^{(n)}\left(t_{2}^{\prime}, t_{3}^{\prime} ; q\right) g\right\rangle_{t_{0}, t_{1}, t_{2}, t_{3} ; q, t}^{(n)}
$$

where $\left(t_{0}^{\prime}, t_{1}^{\prime}, t_{2}^{\prime}, t_{3}^{\prime}\right)=\left(t^{1 / 2} t_{0}, t^{1 / 2} t_{1}, t^{-1 / 2} t_{2}, t^{-1 / 2} t_{3}\right)$, and

$$
\left\langle h I_{t}^{-(n)}\left(t_{0}, t_{1}, t_{2}, t_{3} ; q\right) f\right\rangle_{t^{1 / 2} t_{0}, t^{1 / 2} t_{1}, t^{1 / 2} t_{2}, t^{1 / 2} t_{3} ; q, t}^{(n-1)}=\left\langle f I_{t}^{+(n-1)}(q) h\right\rangle_{t_{0}, t_{1}, t_{2}, t_{3} ; q, t}^{(n)},
$$

for any $B C_{n}$-symmetric polynomials $f$ and $g$, and any $B C_{n-1}$-symmetric polynomial $h$.

PROOF. Simply change order of integration.

REMARK. Note that here we are using the normalized inner product. 
ERIC M. RAINS

COROLlary 3.6. The integral operators act on Koornwinder polynomials as follows.

$$
\begin{gathered}
I_{t}^{+(n)}(q) K_{\lambda}^{(n)}\left(; t_{0}, t_{1}, t_{2}, t_{3} ; q, t\right) \\
=t^{|\lambda| / 2} \prod_{1 \leq i \leq m} \frac{1-t^{n+1-\lambda_{i}^{\prime}} q^{i-1}}{1-t^{n+1} q^{i-1}} \\
K_{\lambda}^{(n+1)}\left(; t^{-1 / 2} t_{0}, t^{-1 / 2} t_{1}, t^{-1 / 2} t_{2}, t^{-1 / 2} t_{3} ; q, t\right) \\
I_{t}^{(n)}\left(t_{0}, t_{1} ; q\right) K_{\lambda}^{(n)}\left(; t_{0}, t_{1}, t_{2}, t_{3} ; q, t\right) \\
=t^{|\lambda| / 2} \prod_{1 \leq i \leq m} \frac{1-t^{n-\lambda_{i}^{\prime}} q^{i-1} t_{0} t_{1}}{1-t^{n} q^{i-1} t_{0} t_{1}} \\
K_{\lambda}^{(n)}\left(; t^{1 / 2} t_{0}, t^{1 / 2} t_{1}, t^{-1 / 2} t_{2}, t^{-1 / 2} t_{3} ; q, t\right) \\
I_{t}^{-(n)}\left(t_{0}, t_{1}, t_{2}, t_{3} ; q\right) K_{\lambda}^{(n)}\left(; t_{0}, t_{1}, t_{2}, t_{3} ; q, t\right) \\
=t^{|\lambda| / 2} \prod_{1 \leq i \leq m} \frac{1-t^{n-1-\lambda_{i}^{\prime}} q^{i-1} t_{0} t_{1} t_{2} t_{3}}{1-t^{n-1} q^{i-1} t_{0} t_{1} t_{2} t_{3}} \\
K_{\lambda}^{(n-1)}\left(; t^{1 / 2} t_{0}, t^{1 / 2} t_{1}, t^{1 / 2} t_{2}, t^{1 / 2} t_{3} ; q, t\right) .
\end{gathered}
$$

REMARK. In particular, note that

$$
D_{q}^{-(n+1)}(t) I_{t}^{+(n)}(q)=I_{t}^{-(n)}\left(t_{0}, t_{1}, t_{2}, t_{3} ; t\right) D_{q}^{+(n)}\left(t_{0}, t_{1}, t_{2}, t_{3} ; t\right)=0 .
$$

Corollary 3.7. For the principal specialization, we have:

$$
\frac{k_{\lambda}^{(n)}\left(t^{1 / 2} t_{0}: t^{1 / 2} t_{1}, t^{-1 / 2} t_{2}, t^{-1 / 2} t_{3} ; q, t\right)}{k_{\lambda}^{(n)}\left(t_{0}: t_{1}, t_{2}, t_{3} ; q, t\right)}=t^{-|\lambda| / 2} \prod_{1 \leq i \leq m} \frac{1-t^{n} q^{i-1} t_{0} t_{1}}{1-t^{n-\lambda_{i}^{\prime}} q^{i-1} t_{0} t_{1}}
$$

and

$$
\frac{k_{\lambda}^{(n+1)}\left(t^{-1 / 2} t_{0}: t^{-1 / 2} t_{1}, t^{-1 / 2} t_{2}, t^{-1 / 2} t_{3} ; q, t\right)}{k_{\lambda}^{(n)}\left(t_{0}: t_{1}, t_{2}, t_{3} ; q, t\right)}=t^{-|\lambda| / 2} \prod_{1 \leq i \leq m} \frac{1-t^{n+1} q^{i-1}}{1-t^{n+1-\lambda_{i}^{\prime}} q^{i-1}}
$$

For the norms of (normalized) Koornwinder polynomials, we have:

$$
\begin{aligned}
& \frac{N_{\lambda}^{(n)}\left(t^{1 / 2} t_{0}: t^{1 / 2} t_{1}, t^{-1 / 2} t_{2}, t^{-1 / 2} t_{3} ; q, t\right)}{N_{\lambda}^{(n)}\left(t_{0}: t_{1}, t_{2}, t_{3} ; q, t\right)} \\
& =t^{|\lambda|} \prod_{1 \leq i \leq m} \frac{\left(1-t^{n-\lambda_{i}^{\prime}} q^{i-1} t_{0} t_{1}\right)\left(1-t^{n-\lambda_{i}^{\prime}} q^{i-1} t_{2} t_{3} / t\right)}{\left(1-t^{n} q^{i-1} t_{0} t_{1}\right)\left(1-t^{n} q^{i-1} t_{2} t_{3} / t\right)}
\end{aligned}
$$

and

$$
\begin{aligned}
& \frac{N_{\lambda}^{(n+1)}\left(t^{-1 / 2} t_{0}: t^{-1 / 2} t_{1}, t^{-1 / 2} t_{2}, t^{-1 / 2} t_{3} ; q, t\right)}{N_{\lambda}^{(n)}\left(t_{0}: t_{1}, t_{2}, t_{3} ; q, t\right)} \\
& =t^{|\lambda|} \prod_{1 \leq i \leq m} \frac{\left(1-t^{n+1-\lambda_{i}^{\prime}} q^{i-1}\right)\left(1-t^{n-\lambda_{i}^{\prime}} q^{i-1} t_{0} t_{1} t_{2} t_{3} / t^{2}\right)}{\left(1-t^{n+1} q^{i-1}\right)\left(1-t^{n} q^{i-1} t_{0} t_{1} t_{2} t_{3} / t^{2}\right)}
\end{aligned}
$$

PROOF. The recurrences for the principal specialization follow from the observation that the limiting integral corresponding to the principal specialization of 
$I_{t}^{+(n)}(; q) f$ or $I_{t}^{(n)}\left(t_{0}, t_{1} ; q\right) f$ resolves (modulo symmetry) into a single residue, and is thus simply the principal specialization of $f$ itself. The result thus follows from the action of these operators on Koornwinder polynomials. The recurrences for the norm follow immediately from adjointness.

\section{The difference-integral representation}

Theorem 4.1. 14, 15. The principal specialization and norm of Koornwinder polynomials are given by the following formulas.

$$
\begin{aligned}
& k_{\lambda}^{(n)}\left(t_{0}: t_{1}, t_{2}, t_{3} ; q, t\right)=\left(t_{0} t^{n-1}\right)^{-|\lambda|} t^{n(\lambda)} \frac{C_{\lambda}^{0}\left(t^{n}, t^{n-1} t_{0} t_{1}, t^{n-1} t_{0} t_{2}, t^{n-1} t_{0} t_{3} ; q, t\right)}{C_{\lambda}^{-}(t ; q, t) C_{\lambda}^{+}\left(t^{2 n-2} t_{0} t_{1} t_{2} t_{3} / q ; q, t\right)} \\
& N_{\lambda}^{(n)}\left(t_{0}: t_{1}, t_{2}, t_{3} ; q, t\right)=\Delta_{\lambda}\left(t^{2 n-2} t_{0} t_{1} t_{2} t_{3} / q \mid t^{n}, t^{n-1} t_{0} t_{1}, t^{n-1} t_{0} t_{2}, t^{n-1} t_{0} t_{3} ; q, t\right)^{-1}
\end{aligned}
$$

PROOF. The recurrences of Corollary 2.5 allow us to deduce the formulas for $\lambda+1^{n}$ from the formula for $\lambda$; it thus suffices to consider the case $\lambda_{n}=0$. But then the recurrences of Corollary 3.7 prove this case, given that the theorem holds in $n-1$ dimensions. Since the theorem holds for $\lambda=0$, it holds in general.

The structure of the above induction gives rise to the following construction of Koornwinder polynomials.

Theorem 4.2. Construct a family $\hat{K}_{\lambda}^{(n)}\left(; t_{0}, t_{1}, t_{2}, t_{3} ; q, t\right)$ of $B C_{n}$-symmetric polynomials, defined for nonnegative integers $n$ and partitions $\lambda$ with $\ell(\lambda) \leq n$, as follows.

(i) $\hat{K}_{0}^{(0)}\left(; t_{0}, t_{1}, t_{2}, t_{3} ; q, t\right)=1$

(ii) For $n>0, \lambda_{n}=0$,

$$
\begin{aligned}
\hat{K}_{\lambda}^{(n)}\left(; t_{0}, t_{1}, t_{2}, t_{3} ; q, t\right)= & t^{-|\lambda| / 2} \prod_{\substack{1 \leq i \leq m\\
}} \frac{1-t^{n} q^{i-1}}{1-t^{n-\lambda_{i}^{\prime}} q^{i-1}} \\
& I_{t}^{+(n-1)}(q) \hat{K}_{\lambda}^{(n-1)}\left(; t^{1 / 2} t_{0}, t^{1 / 2} t_{1}, t^{1 / 2} t_{2}, t^{1 / 2} t_{3} ; q, t\right)
\end{aligned}
$$

(iii) For $n>0, \lambda_{n}>0$,

$$
\begin{aligned}
\hat{K}_{\lambda}^{(n)}\left(; t_{0}, t_{1}, t_{2}, t_{3} ; q, t\right)= & q^{(|\lambda|-n) / 2} \prod_{1 \leq i \leq n}\left(1-q^{\lambda_{i}} t^{n-i} t_{0} t_{1} t_{2} t_{3} / q\right)^{-1} \\
& D_{q}^{+(n)}\left(t_{0}, t_{1}, t_{2}, t_{3} ; t\right) \\
& \hat{K}_{\lambda-1^{n}}^{(n)}\left(; q^{1 / 2} t_{0}, q^{1 / 2} t_{1}, q^{1 / 2} t_{2}, q^{1 / 2} t_{3} ; q, t\right) .
\end{aligned}
$$

Then the resulting polynomials are simply the Koornwinder polynomials.

REMARK 4.3. Similarly, one can define a family of polynomials by

$$
\begin{gathered}
\bar{P}_{\lambda}^{*(n)}(; q, t, s)=t^{-|\lambda| / 2} \prod_{1 \leq i \leq m} \frac{1-t^{n} q^{i-1}}{1-t^{n-\lambda_{i}^{\prime}} q^{i-1}} I_{t}^{+(n-1)}(q) \bar{P}_{\lambda}^{*(n-1)}(; q, t, s) \\
\bar{P}_{\lambda}^{*(n)}\left(x_{1}, x_{2} \ldots x_{n} ; q, t, s\right)=\prod_{\substack{1 \leq i \leq n\\
}}\left(x_{i}+x_{i}^{-1}-s-s^{-1}\right) \\
\bar{P}_{\lambda-1^{n}}^{*(n)}\left(x_{1}, x_{2}, \ldots x_{n} ; q, t, s q\right) .
\end{gathered}
$$


The resulting polynomials are simply (the symmetric versions of) Okounkov's interpolation polynomials [10; see also 11. Indeed, this differs from Okounkov's integral representation for these polynomials only in that our integral operator is defined by a contour integral, rather than a sum. When the polynomial is specialized at a point of the form $q^{\mu_{i}} t^{n-i} s$, our contour integral becomes a sum over partitions by residue calculus, and agrees in that case with Okounkov's $q$-integral. Thus the above construction for Koornwinder polynomials can be viewed as an analogue of Okounkov's representation; in fact, these are both special cases of the construction given in $\mathbf{1 3}$

REMARK 4.4. Unfortunately, the above machinery does not appear to give rise to a similar proof of evaluation symmetry; of course, we can always refer to the arguments of van Diejen [15] or Okounkov [10] showing that evaluation symmetry follows from the principal specialization formula.

Another straightforward consequence of our machinery is the following result of Mimachi.

Theorem 4.5. [8] For any integers $m, n \geq 0$,

$$
\begin{array}{r}
\prod_{\substack{1 \leq i \leq n \\
1 \leq j \leq m}}\left(y_{j}+y_{j}^{-1}-x_{i}-x_{i}^{-1}\right)=\sum_{\lambda \subset m^{n}}(-1)^{|\lambda|} K_{\lambda}^{(n)}\left(x_{1}, \ldots x_{n} ; t_{0}, t_{1}, t_{2}, t_{3} ; q, t\right) \\
K_{n^{m}-\lambda^{\prime}}^{(m)}\left(y_{1}, \ldots y_{m} ; t_{0}, t_{1}, t_{2}, t_{3} ; t, q\right)
\end{array}
$$

Proof. Clearly the left-hand side admits some expansion of the form

$$
\sum_{\lambda, \mu \subset m^{n}} c_{\lambda \mu} K_{\lambda}^{(n)}\left(x_{1}, \ldots x_{n} ; t_{0}, t_{1}, t_{2}, t_{3} ; q, t\right) K_{\mu}^{(m)}\left(y_{1}, \ldots y_{m} ; t_{0}, t_{1}, t_{2}, t_{3} ; t, q\right)
$$

If we apply one of the "second-order" difference operators for which $K_{\lambda}^{(n)}$ is a basis of eigenfunctions, the proof of Theorem 3.4 turns this composition of two difference operators in the $x$ variables into a composition of two integral operators in the $y$ variables, for which $K_{\mu}^{(m)}$ is a basis of eigenfunctions. Comparing the two eigenvalues, we find that $c_{\lambda \mu}=0$ unless $\mu=n^{m}-\lambda^{\prime}$. The coefficient then follows by an examination of dominant terms.

\section{References}

[1] R. Askey and J. Wilson. Some basic hypergeometric orthogonal polynomials that generalize Jacobi polynomials. Number 319 in Memoirs of the AMS. Amer. Math. Soc., Providence, RI, 1985.

[2] O. A. Chalykh. Macdonald polynomials and algebraic integrability. Adv. Math., 166(2):193259, 2002.

[3] I. Cherednik. Double affine Hecke algebras and Macdonald's conjectures. Ann. of Math. (2), 141(1):191-216, 1995.

[4] R. A. Gustafson. A generalization of Selberg's beta integral. Bull. Amer. Math. Soc. (N.S.), 22(1):97-105, 1990.

[5] R. A. Gustafson. Some $q$-beta and Mellin-Barnes integrals on compact Lie groups and Lie algebras. Trans. Amer. Math. Soc., 341(1):69-119, 1994.

[6] T. H. Koornwinder. Askey-Wilson polynomials for root systems of type BC. In Donald St. P. Richards, editor, Hypergeometric functions on domains of positivity, Jack polynomials, and applications (Tampa, FL, 1991), Contemp. Math. 138, pages 189-204. Amer. Math. Soc., Providence, RI, 1992. 
[7] I. G. Macdonald. Affine Hecke algebras and orthogonal polynomials, volume 157 of Cambridge Tracts in Mathematics. Cambridge University Press, Cambridge, 2003.

[8] K. Mimachi. A duality of Macdonald-Koornwinder polynomials and its application to integral representations. Duke Math. J., 107(2):265-281, 2001.

[9] M. Noumi. Macdonald-Koornwinder polynomials and affine Hecke rings. Sūrikaisekikenkyūsho Kōkyūroku, (919):44-55, 1995. Various aspects of hypergeometric functions (Japanese) (Kyoto, 1994).

[10] A. Okounkov. BC-type interpolation Macdonald polynomials and binomial formula for Koornwinder polynomials. Transform. Groups, 3(2):181-207, 1998.

[11] E. M. Rains. $B C_{n}$-symmetric polynomials. arxiv:math.QA/0112035

[12] E. M. Rains. $B C_{n}$-symmetric theta functions. arxiv:math.CO/0402113

[13] E. M. Rains. Transformations of elliptic hypergeometric integrals. arxiv:math.QA/0309252

[14] S. Sahi. Nonsymmetric Koornwinder polynomials and duality. Ann. of Math. (2), 150(1):267282, 1999.

[15] J. F. van Diejen. Self-dual Koornwinder-Macdonald polynomials. Invent. Math., 126(2):319$339,1996$.

Department of Mathematics, University of California, Davis

E-mail address: rains@math.ucdavis.edu 